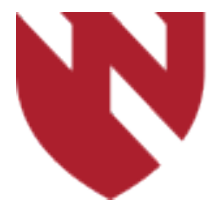

December 2020

\title{
Multi-digit Auto-amputation in Setting of Mixed Connective Tissue Disease
}

\author{
Kishan P. Patel \\ University of Nebraska Medical Center \\ Jennifer L. Medlin \\ University of Nebraska Medical Center \\ Michelene Hearth-Holmes \\ University of Nebraska Medical Center
}

Tell us how you used this information in this short survey.

Follow this and additional works at: https://digitalcommons.unmc.edu/gmerj

Part of the Higher Education Commons, Musculoskeletal Diseases Commons, Rheumatology

Commons, and the Skin and Connective Tissue Diseases Commons

\section{Recommended Citation}

1. Fardoun MM, Nassif J, Issa K, Baydoun E, Eid AH. Raynaud's Phenomenon: A Brief Review of the Underlying Mechanisms. Frontiers in Pharmacology. 2016;7 2. Wigley FM, Flavahan NA. Raynaud's phenomenon. N Engl J Med 2016; 375:556-565 3. Harrison BJ, Silman AJ, Hider SL, Herrick AL. Cigarette smoking: a significant risk factor for digital vascular diseases in patients with systemic sclerosis. Arthritis Rheum 2002;46:3312-6 4. Landry GJ. Current medical and surgical management of Raynauds syndrome. Journal of Vascular Surgery. 2013;57:1710-6. 5. Abou-Raya A, Abou-Raya S, Helmii M. Statins: potentially useful in therapy of systemic sclerosis-related Raynaud's phenomenon and digital ulcers. J Rheumatol 2008; 35:1801-1808

This Case Report is brought to you for free and open access by DigitalCommons@UNMC. It has been accepted for inclusion in Graduate Medical Education Research Journal by an authorized editor of DigitalCommons@UNMC. For more information, please contact digitalcommons@unmc.edu. 


\title{
Multi-digit Auto-amputation in Setting of Mixed Connective Tissue Disease
}

\author{
Abstract \\ Raynaud's disease is a cutaneous manifestation often present in various connective tissue and vascular \\ diseases. Depending on the age of onset and acuity of symptoms, the phenomenon can provide clinicians \\ with some insight as to the severity of underlying disease, including the distinction between primary and \\ secondary Raynaud's. However, this diagnostic approach seems to have its limitations regarding efficacy \\ and timing of treatment. Traditionally, treatment of Raynaud's has focused on reestablishing blood \\ perfusion to affected tissue, which has previously been achieved with vasodilatory medications and site- \\ specific surgical intervention. In lieu of the severe sequelae of Raynaud's in the setting of mixed \\ connective tissue disease, including autoamputation, preemptive treatment of Raynaud's should be \\ considered, as distinguishing between a primary and secondary etiology of Raynaud's can often be \\ difficult to discern. This case highlights the importance of conventional salvage therapies prior to \\ establishing a definitive diagnosis or etiology of the phenomenon, as evidenced by our patient \\ experiencing irreversible ischemic damage resulting in uncontrolled autoamputation of her digits.

\section{Keywords} \\ Mixed connective tissue disease; auto-amputation; ischemia; Raynaud's disease; thromboangiitis \\ obliterans

\section{Creative Commons License} \\ (c) (i) (9) \\ This work is licensed under a Creative Commons Attribution-Noncommercial-No Derivative Works 4.0 \\ License.
}




\section{Multi-Digit Auto-Amputation in Setting of Mixed Connective Tissue Disease \\ Kishan P. Patel', Jennifer L. Medlin², Michelene Hearth-Holmes²}

${ }^{1}$ University of Nebraska Medical Center, Department of Internal Medicine, Omaha, NE

${ }^{2}$ University of Nebraska Medical Center, Department of Internal Medicine, Division of Rheumatology, Omaha, NE

\begin{abstract}
Raynaud's disease is a cutaneous manifestation often present in various connective tissue and vascular diseases. Depending on the age of onset and acuity of symptoms, the phenomenon can provide clinicians with some insight as to the severity of underlying disease, including the distinction between primary and secondary Raynaud's. However, this diagnostic approach seems to have its limitations regarding efficacy and timing of treatment. Traditionally, treatment of Raynaud's has focused on reestablishing blood perfusion to affected tissue, which has previously been achieved with vasodilatory medications and site-specific surgical intervention. In lieu of the severe sequelae of Raynaud's in the setting of mixed connective tissue disease, including autoamputation, preemptive treatment of Raynaud's should be considered, as distinguishing between a primary and secondary etiology of Raynaud's can often be difficult to discern. This case highlights the importance of conventional salvage therapies prior to establishing a definitive diagnosis or etiology of the phenomenon, as evidenced by our patient experiencing irreversible ischemic damage resulting in uncontrolled autoamputation of her digits.
\end{abstract}

\section{Introduction}

Raynaud's Disease is a common vascular physiologic response which typically manifests as distal digit skin changes and rapid skin demarcation in up to $10 \%$ of the general population. ${ }^{1}$ In primary Raynaud's, a defect in the sympathetic signaling response affects local vasculature. When subjected to cold temperatures or external stressors, the distal arteries and cutaneous arterioles abnormally constrict, causing compromised blood flow to the affected anatomy. ${ }^{1,2}$ In a clinical setting, greater than $80 \%$ of those presenting with Raynaud's demonstrate findings consistent with primary disease. ${ }^{2}$ As such, reversing the stressor typically allows for vasodilation of the affected arteries resulting in symptomatic relief within 15-20 minutes time. ${ }^{1}$

In secondary forms of the condition, also known as Raynaud's Phenomenon, the normal vascular physiology can be disrupted as an insult due to, but not limited to, systemic disease, medications, social history factors, trauma, elevated estrogen levels, carpal tunnel syndrome, intravenous drug use, and tobacco abuse. ${ }^{1-3}$ Despite the absence of a specific FDA-approved treatment, Raynaud's can be well controlled through concurrent vasodilatory medications, preventive measures, behavioral therapy, and stressor avoidance. ${ }^{4}$ In severe cases, individuals with prolonged ischemia can develop distal ulcers and gangrenous tissue. ${ }^{1}$ Digital ulceration can be prevented by improving endothelial function. In very rare cases, the distal aspects of the affected limbs may experience irreversible damage requiring amputation or surgical intervention. ${ }^{4}$ Herein, we present a case of multi-digit autoamputation having obtained formal consent by the patient for educational purposes.

\section{Case Presentation}

A 54-year-old female with a history of undifferentiated connective tissue disease, inflammatory polyarthritis, hypertension, Hashimoto's thyroiditis, and nicotine use disorder presented with severe, diffuse, recurring joint pain and progressively worsening morning stiffness. Initially, she was suspected to have an inflammatory arthritis flare and given a course of steroids. In the weeks to follow, she developed bilateral cyanotic changes in her fingertips, splinter hemorrhages, and metacarpophalangeal joint nodules. A transthoracic echocardiogram did not reveal signs of an embolic endocarditis nor were there any signs of an infectious etiology present. Her distal digits remained cyanotic and painful, requiring the use of amlodipine and sildenafil to restore blood perfusion. In addition, she developed an intolerance to air-conditioned climates, multiple telangiectasias, and intermittent dysphagia to solid foods.

Laboratory testing confirmed the presence of ribonucleoprotein (anti-U1RNP) antibody, smooth-muscle antibody, and mitochondrial antibody. Rheumatoid factor, anti-Sjögren'ssyndrome (SS) related antigen A (antiRo), anti-SS related antigen B (anti-La), anti-topoisomerase I (anti-Scl-70), anticentromere, anti-histidyl-tRNA (JO-1), anti-Ribonucleic acid polymerase (antiRNA Pol III), anti-neutrophil cytoplasmic antibodies (ANCA), myeloperoxidase ANCA (MPO-ANCA), Proteinase 3 ANCA (PR3-ANCA), lupus anticoagulant, and anticardiolipin antibodies were all negative.
Based on serological testing, her presentation was thought to be more suggestive of a combined mixed connective tissue disease and thromboangiitis obliterans rather than scleroderma or systemic sclerosis. Her ischemia related pains progressively worsened to the extent that digital blockade with local anesthesia was required, though only providing limited relief. A computed tomography angiography of the chest and upper extremities demonstrated patency, with adequately perfused blood flow to her subclavian arteries and distal vessels to the level of the bilateral wrists. Meanwhile, the patient began to develop ulcerations on her distal digits, for which a digital sympathectomy was recommended, although deferred by the patient. Multiple digits began to auto-amputate (Figure 1). As a consequence, compromised blood flow to the distal portions of her digits caused chronic osteomyelitic changes requiring surgical intervention (Figures. 2-3).

\section{Discussion}

Raynaud's disease is characterized by temperature-induced vasoconstriction, leading to vasospastic attacks associated with the classic triple-color change, along with puffiness and ulcerations predominantly at the level of the digits. ${ }^{1}$ The defect in primary Raynaud's lies within the affected vasculature and sympathetic signaling response; in secondary Raynaud's the vascular physiology can be disrupted due to systemic disease, tobacco abuse, etc. ${ }^{2}$

Auto amputation is an unlikely sequela of Raynaud's despite clinical intervention. It was probable that this patient's ischemic changes were multifactorial in nature including mixed connective tissue disease (MCTD), atherosclerosis, tobacco abuse, and thromboangiitis obliterans. An extensive smoking history can increase the probability of a digital vascular complication by up to $400 \%$ in patients diagnosed with systemic sclerosis, which may have been a contributing factor in our presenting patient. ${ }^{3}$ Our patient required vasodilatory medications, with both a calcium channel blocker and a phosphodiesterase V inhibitor. She participated in behavioral therapy and was successful in cold temperature avoidance and smoking cessation. Furthermore, cholesterol modifying drugs have been shown to improve limited patient outcomes in 84 cases of 
systemic sclerosis, with a majority of cases demonstrating a significant reduction of digital ulcerations on maintenance statins. ${ }^{5}$

Ultimately, the patient required percutaneous anesthetic digital injections, as well as bilateral sympathectomy prior to regaining control and entering remission two years following disease onset. Cervicothoracic sympathectomy is typically a rare intervention reserved for patients experiencing severe recurrence. The procedure is significantly effective in pain management, whereas digital arterial sympathectomy has less evidence of success in controlled trials. ${ }^{4}$

\section{Conclusion}

Traditionally, treatment of Raynaud's disease has focused on reestablishing blood perfusion to affected tissue, which has previously been achieved with vasodilatory medications and site-specific surgical intervention. Patients with underlying autoimmune disorders and/ or consistent serologies, including those of MCTD, especially if they are smokers, should be monitored closely and early initiation of vasodilators should be considered to prevent complications. This case highlights the importance of conventional salvage therapies prior to establishing a definitive diagnosis or etiology of the phenomenon, as evidenced by our patient experiencing irreversible ischemic damage resulting in uncontrolled autoamputation of her digits. Preemptive treatment of Raynaud's should be considered, as distinguishing between a primary and secondary etiology of Raynaud's can often be difficult to discern.

https://doi.org/10.32873/unmc.dc.gmerj.2.2.011

\section{References}

1 Fardoun MM, Nassif J, Issa K, Baydoun E, Eid AH. Raynaud's Phenomenon: A Brief Review of the Underlying Mechanisms. Frontiers in Pharmacology. 2016;7

2 Wigley FM, Flavahan NA. Raynaud's phenomenon. N Engl J Med 2016; 375:556-565

3 Harrison BJ, Silman AJ, Hider SL, Herrick AL. Cigarette smoking: a significant risk factor for digital vascular diseases in patients with systemic sclerosis. Arthritis Rheum 2002;46:3312-6

4 Landry GJ. Current medical and surgical management of Raynauds syndrome. Journal of Vascular Surgery. 2013;57:1710-6.

5 Abou-Raya A, Abou-Raya S, Helmii M. Statins: potentially useful in therapy of systemic sclerosis-related Raynaud's phenomenon and digital ulcers. J Rheumatol 2008; 35:18011808 .

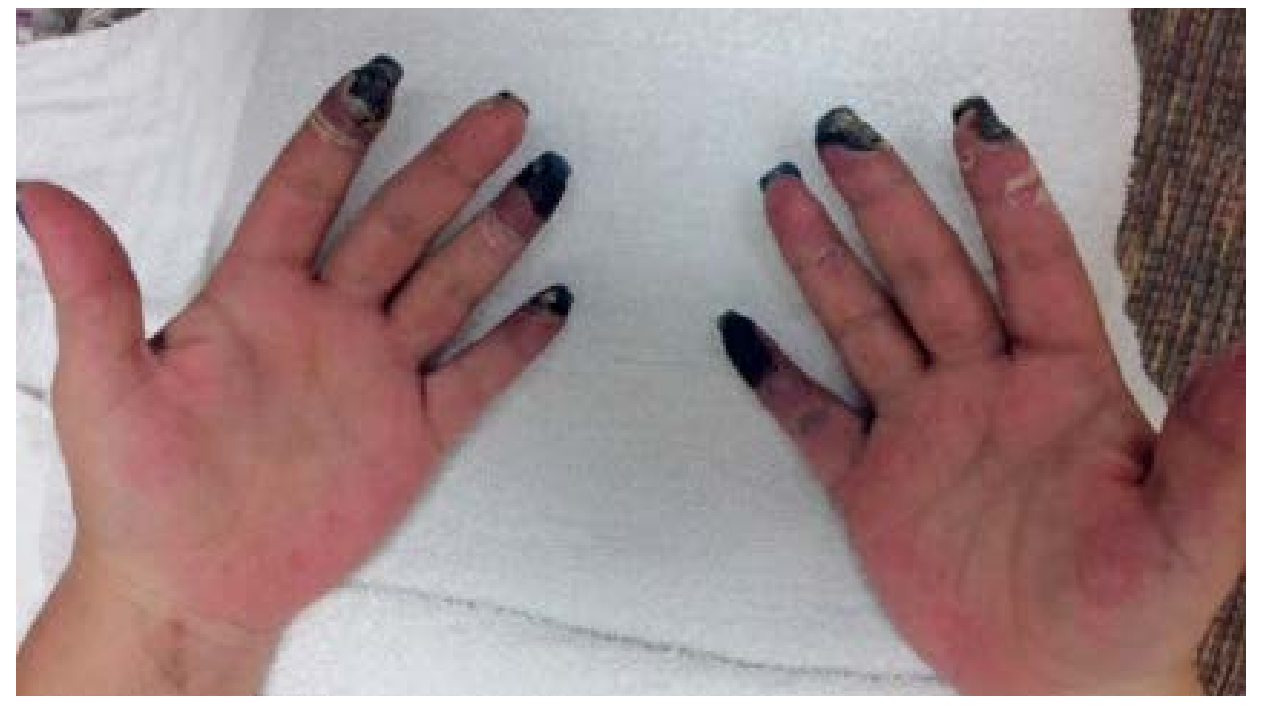

Figure 1. Bilateral hands with distal digit ulceration and cyanotic changes.

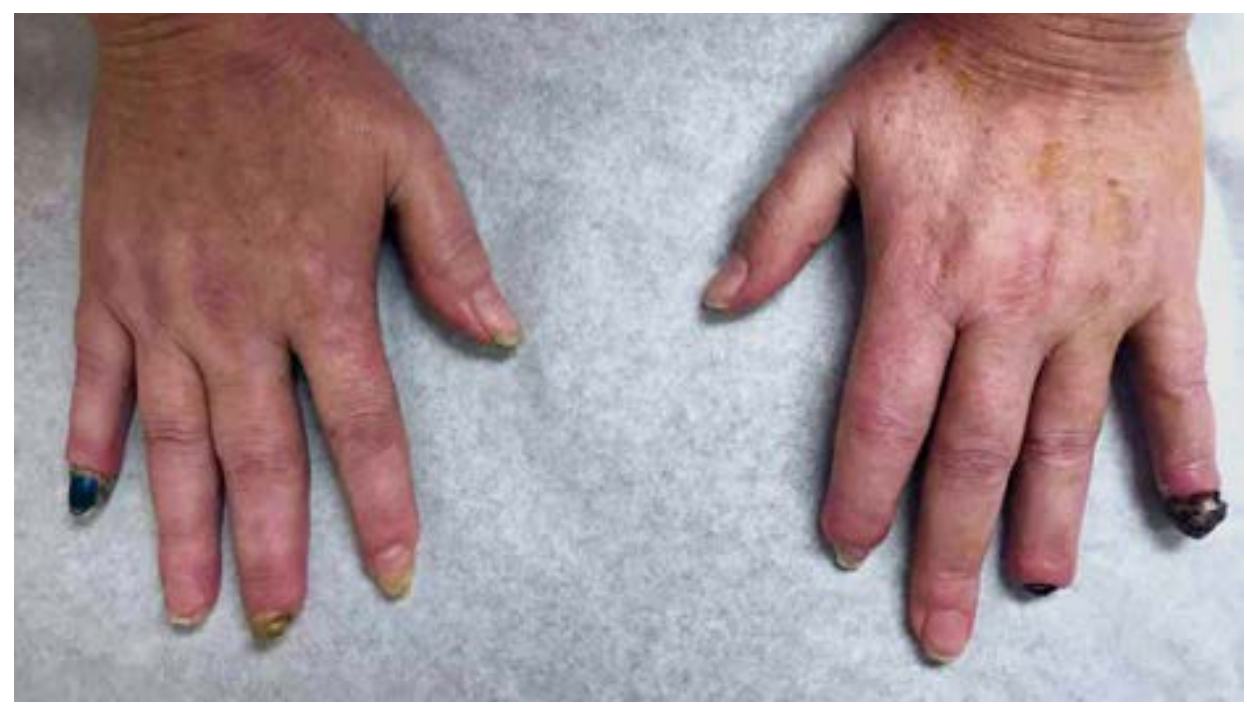

Figure 2. Bilateral hands with chronic osteomyelitic changes post-surgical intervention (dorsal aspect of hands).

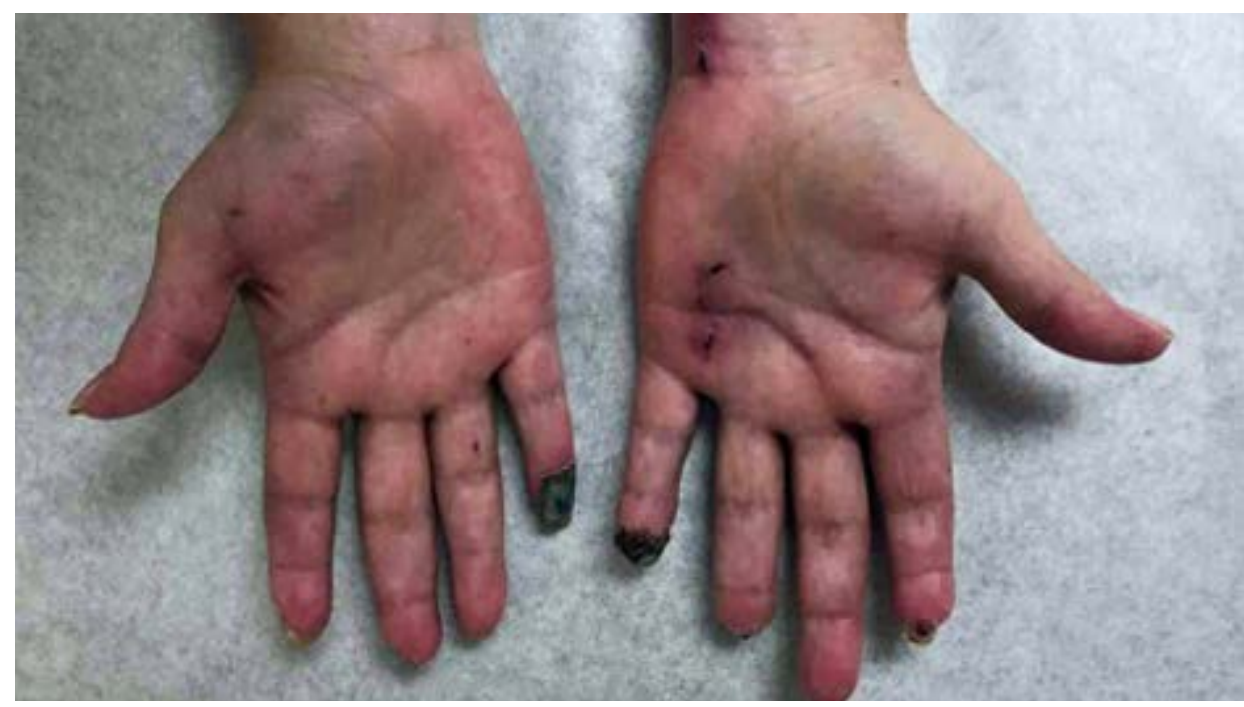

Figure 3. Bilateral hands with chronic osteomyelitic changes post-surgical intervention (palmar aspect of hands). 